\title{
Angiopoietin-like protein 2 as a potential biomarker for colorectal cancer
}

\author{
TAKUMA YOSHINAGA ${ }^{1,2}$, TAKAMASA SHIGEMITSU ${ }^{2}$, HIROTO NISHIMATA $^{3}$, MASAKI KITAZONO ${ }^{4}$, \\ EMIKO HORI $^{1}$, AYAKO TOMIYOSHI ${ }^{1}$, TAKAYUKI TAKEI ${ }^{2}$ and MASAHIRO YOSHIDA ${ }^{2}$ \\ ${ }^{1}$ Division of Clinical Application, Nanpuh Hospital, Kagoshima 891-8512; ${ }^{2}$ Department of Chemical Engineering, \\ Graduate School of Science and Engineering, Kagoshima University, Kagoshima 890-0065; Departments \\ of ${ }^{3}$ Gastroenterology and ${ }^{4}$ Gastrointestinal Surgery, Nanpuh Hospital, Kagoshima 891-8512, Japan
}

Received February 6, 2015; Accepted April 15, 2015

DOI: $10.3892 / \mathrm{mco} .2015 .577$

\begin{abstract}
Colorectal cancer (CRC) is the third most common malignancy worldwide. Disease progression leads to its spread to other organs, such as the liver, and is associated with higher mortality rates. Early CRC detection is therefore crucial for maximizing the chances of complete cure. The measurement of serum-based tumor biomarkers has shown great potential for the detection of CRC. In this study, we investigated the feasibility of using angiopoietin-like protein 2 (ANGPTL2) as a candidate biomarker for CRC. We first investigated ANGPTL2 expression in 7 CRC cell lines, among which Colo320, NCC-CoCK-115P, Caco-2 and Colo205 exhibited comparatively high ANGPTL2 expression. The serum levels of ANGPTL2 in CRC patients $(3.45 \pm 1.30 \mathrm{ng} / \mathrm{ml})$ were higher compared with those in healthy controls $(2.74 \pm 0.64 \mathrm{ng} / \mathrm{ml})$ $(\mathrm{P}<0.05)$. A receiver operating characteristic analysis demonstrated that the diagnostic performance of ANGPTL2 was marginally lower compared with that of the established biomarker C-reactive protein, but higher compared with that of carbohydrate antigen 19-9. These results suggested that the simultaneous measurement of ANGPTL2, along with previously established serum biomarkers, may increase the likelihood of early detection of CRC.
\end{abstract}

\section{Introduction}

Colorectal cancer (CRC) is the third most common malignancy worldwide, with 1.2 million new diagnoses and $>0.8$ million CRC-related deaths worldwide annually. Disease progression results in metastasis to other organs, such as the liver, and is associated with higher mortality rates (1). The likelihood

Correspondence to: Professor Masahiro Yoshida, Department of Chemical Engineering, Graduate School of Science and Engineering, Kagoshima University, 1-21-40 Korimoto, Kagoshima 890-0065, Japan

E-mail: myoshida@cen.kagoshima-u.ac.jp

Key words: angiopoietin-like protein 2, colorectal cancer, serum, biomarker, mucinous carcinoma of successful complete excision is therefore significantly increased if surgery is performed during the early stages. Thus, early detection of CRC is crucial for maximizing the chances of complete cure (2). The measurement of serum-based tumor biomarkers is a promising screening method for the detection of CRC. C-reactive protein (CRP) and carbohydrate antigen 19-9 (CA19-9) are among the most commonly used serum-based tumor-associated antigens in the management of CRC patients $(3,4)$.

In this study, we investigated the potential usefulness of angiopoietin-like protein 2 (ANGPTL2) as a candidate biomarker for CRC. ANGPTL2 is a secreted protein that regulates angiogenesis in vivo (5). Angiogenic factors produced by tumor cells play an important role in tumor growth. Additionally, this protein is reported to be a regulatory factor of chronic inflammation (6-10). Endo et al (9) reported that ANGPTL2 is a promising biomarker for diagnosing human lung and breast cancers. Those reports raised the possibility that ANGPTL2 may also be a candidate biomarker for other types of cancer. We previously demonstrated that ANGPTL2 is upregulated in a gastric cancer cell line and in gastric cancer patients, demonstrating that this protein is a clinically useful biomarker for gastric cancer (11). However, the diagnostic usefulness of ANGPTL2 in CRC has not yet been investigated. In this study, we first examined the expression of ANGPTL2 in 7 CRC cell lines, namely Caco-2, LoVo, WiDr, Colo320, Colo205, CW-2 and NCC-CoC-K115P. Subsequently, we compared the ANGPTL2 concentrations in the serum of CRC patients and healthy individuals to evaluate the sensitivity and specificity of this protein as a predictive biomarker for CRC.

\section{Materials and methods}

Cells. A total of 7 human CRC cell lines, namely Caco-2, LoVo, WiDr, Colo320, Colo205, CW-2 and NCC-CoC-K115P) were purchased from the RIKEN BioResource Center (Tsukuba, Ibaraki, Japan) and the Japanese Collection of Research Bioresources Cell Bank (Ibaraki, Osaka, Japan). Caco-2 cells were cultured in minimum essential medium with $0.1 \mathrm{mmol} / \mathrm{l}$ non-essential amino acid solution, $20 \%$ (w/v) fetal bovine serum (FBS), $100 \mathrm{U} / \mathrm{ml}$ penicillin and $100 \mu \mathrm{g} / \mathrm{ml}$ streptomycin at $37^{\circ} \mathrm{C}$ in $5 \% \mathrm{CO}_{2}$. LoVo cells were cultured in 
Ham's F-12 medium with $10 \%$ (v/v) FBS, $100 \mathrm{U} / \mathrm{ml}$ penicillin and $100 \mu \mathrm{g} / \mathrm{ml}$ streptomycin. WiDr cells were cultured in Dulbecco's modified Eagle's medium with $5 \mathrm{mmol} / \mathrm{l} \mathrm{HEPES}$, $10 \%(\mathrm{v} / \mathrm{v}) \mathrm{FBS}, 100 \mathrm{U} / \mathrm{ml}$ penicillin and $100 \mu \mathrm{g} / \mathrm{ml}$ streptomycin. NCC-CoC-K115P cells were cultured in RPMI-1640 medium with $20 \%(\mathrm{v} / \mathrm{v}) \mathrm{FBS}, 100 \mathrm{U} / \mathrm{ml}$ penicillin and $100 \mu \mathrm{g} / \mathrm{ml}$ streptomycin. Colo320, Colo205 and CW-2 cells were cultured in RPMI-1640 medium with $10 \%$ (v/v) FBS, $100 \mathrm{U} / \mathrm{ml}$ penicillin and $100 \mu \mathrm{g} / \mathrm{ml}$ streptomycin.

Cell culture. The 7 human CRC cell lines were seeded in 6 -well plates $\left(6 \times 10^{5}\right.$ cells/well) and incubated at $37^{\circ} \mathrm{C}$ in $5 \% \mathrm{CO}_{2}$. The media in the wells were changed daily. The cells were collected from the wells every day by trypsinization and the cell number was determined using a hemocytometer. Prior to the analysis, samples of medium from cultured cells were stored at $-80^{\circ} \mathrm{C}$.

Serum samples. Serum samples were obtained from 56 participants who attended the clinic between May, 2013 and February, 2014 at the Nanpuh Hospital (Kagoshima, Japan). The participants included 15 patients with CRC [mean age, 63.8 years, standard deviation (SD), 9.4 years] and 41 healthy controls with normal mucosa (mean age, 47.7 years; $\mathrm{SD}, 9.7$ years). Of the $15 \mathrm{CRC}$ patients, 14 were diagnosed with adenocarcinoma and 1 with mucinous adenocarcinoma. The patient group included 6 patients diagnosed with clinical stage 0-I, 6 patients with clinical stage II and 3 patients with clinical stage III disease. Cancer staging was based on a routine histopathological analysis and clinical assessment, according to the tumor-node-metastasis classification. Tumors were classified according to the recommendations of the 5th International Union Against Cancer. The characteristics of the subjects are summarized in Table I. Informed consent was obtained from all the participants. The study design was approved by the Ethics Committee of Nanpuh Hospital, Kagoshima Kyosaikai, Public Interest Inc. Association, Japan. Clinical examinations were performed according to the principles of the Declaration of Helsinki.

Measurement of biomarkers in cell culture media and serum. The concentrations of ANGPTL2 in human serum and cell culture medium samples were determined using an ANGPTL2 enzyme-linked immunosorbent assay kit (Immuno-Biological Laboratories, Co., Ltd., Gunma, Japan). The concentrations of CRP in the serum were determined by latex agglutination using BM6050 (Kyowa Medex, Co., Ltd., Tokyo, Japan) in accordance with the manufacturer's instructions. The concentrations of CA19-9 in the serum were determined using the electrochemiluminescence immunoassay with LUMIPULSE G1200 ${ }^{\circledR}$ (Fujirebio, Co., Ltd., Tokyo, Japan) in accordance with the manufacturer's instructions.

Statistical analysis. The correlation of the serum ANGPTL2 concentration with the patients' age and tumor size was analyzed using Pearson's correlation analysis. The correlation of the serum ANGPTL2 concentration with the degree of differentiation and depth of tumor invasion were analyzed using Spearman's rank correlation analysis. The statistical difference between ANGPTL2 concentration in the serum of
Table I. Characteristics of the subjects.

\begin{tabular}{|c|c|c|c|}
\hline Characteristics & $\begin{array}{c}\text { CRC } \\
\text { patients } \\
(n=15)\end{array}$ & $\begin{array}{l}\text { Healthy } \\
\text { controls } \\
(n=41)\end{array}$ & $\begin{array}{l}\text { Total } \\
(n=56)\end{array}$ \\
\hline \multicolumn{4}{|l|}{ Age, years } \\
\hline Mean \pm SD & $63.8 \pm 9.4$ & $47.7 \pm 9.7$ & $52.0 \pm 11.9$ \\
\hline Range & $44-80$ & $35-75$ & $35-80$ \\
\hline \multicolumn{4}{|l|}{ Gender } \\
\hline Male & 13 & 22 & 35 \\
\hline Female & 2 & 19 & 21 \\
\hline \multicolumn{4}{|l|}{ BMI $\left(\mathrm{kg} / \mathrm{m}^{2}\right)$} \\
\hline Mean \pm SD & $23.2 \pm 3.4$ & $22.1 \pm 2.7$ & $22.4 \pm 2.9$ \\
\hline Range & $16.8-29.0$ & $17.4-29.5$ & $16.8-29.5$ \\
\hline \multicolumn{4}{|l|}{ Tumor stage } \\
\hline $0-\mathrm{I}$ & 6 & - & 6 \\
\hline II & 6 & - & 6 \\
\hline III & 3 & - & 3 \\
\hline \multicolumn{4}{|l|}{$\begin{array}{l}\text { Depth of } \\
\text { invasion }\end{array}$} \\
\hline M & 3 & - & 3 \\
\hline SM & 0 & - & 0 \\
\hline MP & 3 & - & 3 \\
\hline SS/A & $3 / 1$ & - & $3 / 1$ \\
\hline SE/SI & $4 / 1$ & - & $4 / 1$ \\
\hline \multicolumn{4}{|l|}{$\begin{array}{l}\text { Degree of } \\
\text { differentiation }\end{array}$} \\
\hline $\begin{array}{l}\text { Moderate } \\
\text { to poor }\end{array}$ & 2 & - & 2 \\
\hline $\begin{array}{l}\text { High to } \\
\text { moderate }\end{array}$ & 5 & - & 5 \\
\hline High & 8 & - & 8 \\
\hline
\end{tabular}

CRC, colorectal cancer; SD, standard deviation; BMI, body mass index; M, mucosa; SM, submucosa; MP, tunica muscularis propria; SS/A, subserosa/adventitia; SE/SI, serosa exposed/serosa infiltrating.

CRC patients and healthy individuals was analyzed using the rank, non-parametric, statistical Mann-Whitney U test. Data are presented as means \pm SD. A receiver operating characteristic (ROC) curve was constructed to evaluate the diagnostic performance of ANGPTL2 concentration in differentiating between $\mathrm{CRC}$ patients and healthy individuals. $\mathrm{P}<0.05$ was considered to indicate a statistically significant difference.

\section{Results}

ANGPTL2 expression in CRC cell lines. We first investigated ANGPTL2 expression in 7 CRC cell lines. The ANGPTL2 expression rates of the cell lines at the second day of cell culture are presented in Fig. 1. The expression rate in Caco-2, Colo320, Colo205 and NCC-CoC-K115P cells was $0.57 \pm 0.04 \times 10^{-6}$, $2.92 \pm 0.36 \times 10^{-6}, 0.16 \pm 0.06 \times 10^{-6}$ and $1.08 \pm 0.07 \times 10^{-6} \mathrm{ng} / \mathrm{cell} /$ day. LoVo, WiDr and CW-2 cell lines exhibited very low expression of ANGPTL2. Thus, Caco-2, Colo320, Colo205 


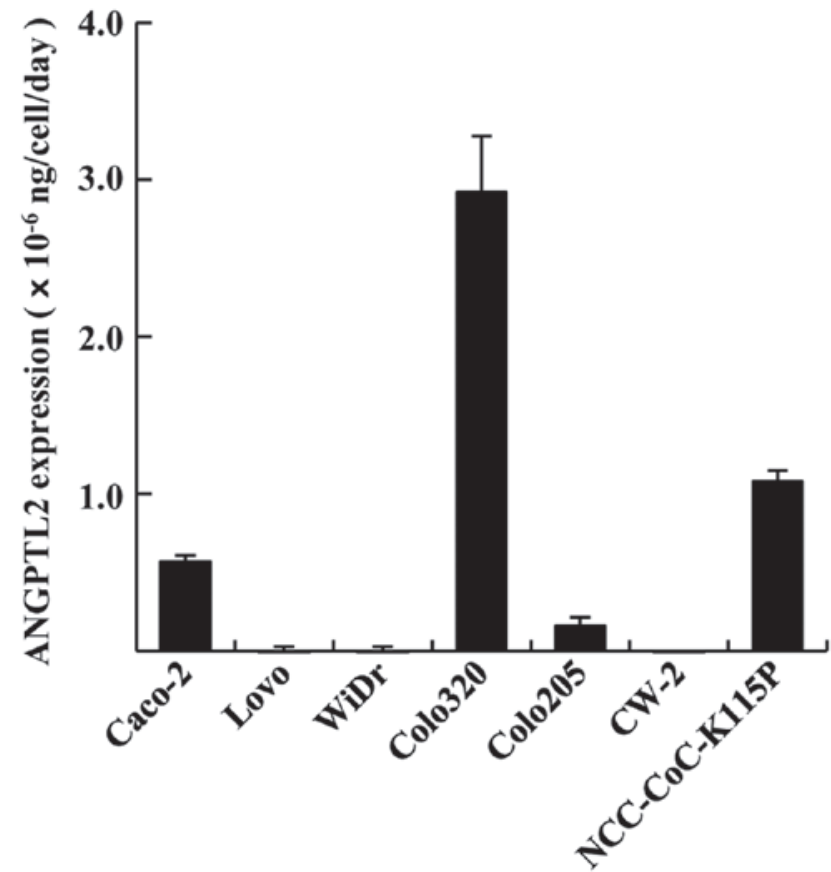

Figure 1. Angiopoietin-like protein 2 (ANGPTL2) expression in 7 colorectal cancer $(\mathrm{CRC})$ cell lines at day 2 of cell culture $(\mathrm{n}=3)$. The error bars indicate standard deviation. The Caco-2, Colo320, Colo205 and NCC-CoC-K115P cell lines exhibited comparatively high ANGPTL2 production.

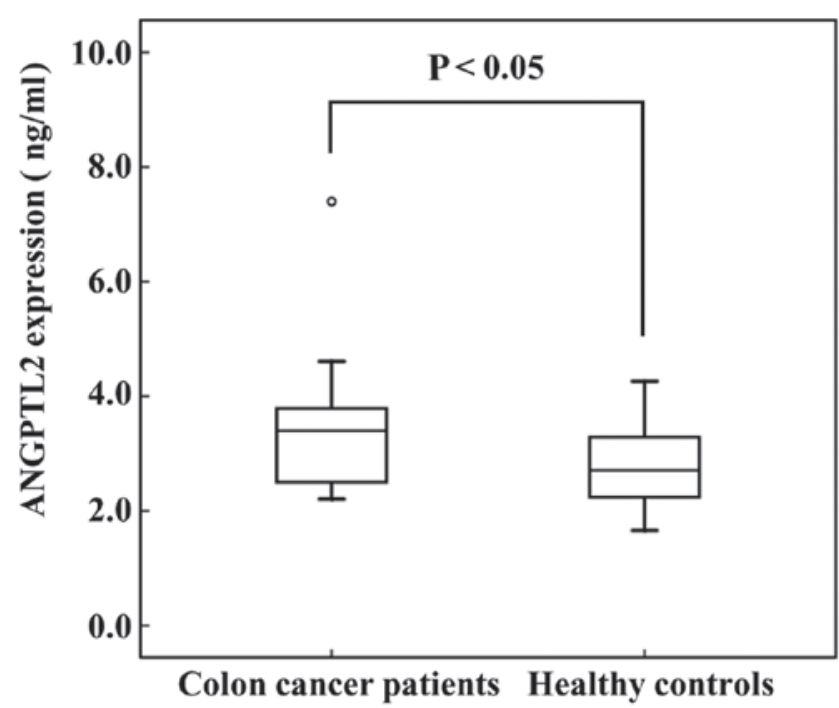

Figure 2. Angiopoietin-like protein 2 (ANGPTL2) concentration in the serum of colorectal cancer $(\mathrm{CRC})$ patients $(\mathrm{n}=15)$ and healthy controls $(n=41)$. The error bars indicate standard deviation. The serum levels of ANGPTL2 in CRC patients were higher compared with those in healthy controls ( $3.45 \pm 1.30$ vs. $2.74 \pm 0.64 \mathrm{ng} / \mathrm{ml}$, respectively) $(\mathrm{P}<0.05)$.

and NCC-CoC-K115P cells exhibited comparatively high ANGPTL2 production. Subsequently, we evaluated ANGTPL2 levels in the serum of CRC patients. The serum levels of ANGPTL2 in CRC patients were higher compared with those in healthy controls $(3.45 \pm 1.30$ vs. $2.74 \pm 0.64 \mathrm{ng} / \mathrm{ml}$, respectively; Fig. 2).

Correlation between serum ANGPTL2 concentration and different variables in CRC patients. There was no correlation
Table II. Correlation between serum ANGPTL2 concentration and different variables in CRC patients $(\mathrm{n}=15)$.

\begin{tabular}{lcc}
\hline Variables & $\begin{array}{c}\text { Correlation } \\
\text { coefficient }\end{array}$ & P-value \\
\hline Age, years & -0.034 & 0.903 \\
Tumor size & -0.029 & 0.924 \\
Degree of differentiation & 0.069 & 0.806 \\
Depth of invasion & 0.327 & 0.234 \\
\hline
\end{tabular}

ANGPTL2, angiopoietin-like protein 2; CRC, colorectal cancer.

Table III. Summary of ROC curve analysis for ANGPTL2, CRP and CA19-9.

\begin{tabular}{lcccc}
\hline Biomarkers & AUC & SE & P-value & $95 \%$ CI \\
\hline $\begin{array}{l}\text { Total data } \\
\text { (stage 0-III) }\end{array}$ & & & & \\
$\quad$ ANGPTL2 & 0.691 & 0.083 & $0.030^{\text {a }}$ & $0.529-0.853$ \\
CRP & 0.763 & 0.087 & $0.003^{\text {a }}$ & $0.591-0.934$ \\
CA19-9 & 0.630 & 0.085 & 0.139 & $0.463-0.797$ \\
Stage $\geq$ II & & & & \\
ANGPTL2 & 0.801 & 0.086 & $0.005^{\text {b }}$ & $0.632-0.970$ \\
CRP & 0.911 & 0.064 & $<0.005^{\mathrm{b}}$ & $0.784-1.037$ \\
CA19-9 & 0.598 & 0.106 & 0.363 & $0.390-0.805$ \\
\end{tabular}

${ }^{\mathrm{a}} \mathrm{P}<0.05 ;{ }^{\mathrm{b}} \mathrm{P}<0.01$. ROC, receiver operating characteristic; ANGPTL2, angiopoietin-like protein 2; CRP, C-reactive protein; CA19-9, carbohydrate antigen 19-9; AUC, area under the curve; SE, standard error; $\mathrm{CI}$, confidence interval.

between the ANGPTL2 level and patient age ( $\mathrm{r}=-0.034$, $\mathrm{P}=0.903)$, tumor size $(\mathrm{r}=-0.029, \mathrm{P}=0.924)$, degree of tumor differentiation $(\mathrm{r}=0.069, \mathrm{P}=0.806)$, or depth of tumor invasion $(\mathrm{r}=0.327, \mathrm{P}=0.234)$ (Table II). We further evaluated the potential of ANGPTL2 as a biomarker of CRC by employing an ROC analysis (Fig. 3, Table III). The area under the ROC curve (AUC) for ANGPTL2 was $0.691[\mathrm{P}=0.030,95 \%$ confidence interval (CI): 0.529-0.853], the AUC for CRP was 0.763 $(\mathrm{P}=0.003$, 95\% CI: 0.591-0.934) and the AUC for CA19-9 was $0.630(\mathrm{P}=0.139,95 \% \mathrm{CI}: 0.463-0.797)$. Additionally, we performed the same ROC analysis in CRC patients with stage II and III tumors (Fig. 3B, Table III). The AUC for ANGPTL2 was 0.801 ( $\mathrm{P}=0.005,95 \%$ CI: $0.632-0.970)$, the AUC for CRP was $0.911(\mathrm{P}<0.005$, 95\% CI: 0.784-1.037) and the AUC for CA19-9 was 0.598 ( $\mathrm{P}=0.363,95 \%$ CI: 0.390-0.805) (Table III). Tumor samples from CRC patients who exhibited the highest ANTPTL2 level were positive for mucin 2 and mucin 5AC (Fig. 4).

\section{Discussion}

To the best of our knowledge, this is the first reported investigation of ANGPTL2 expression in CRC. The Colo320 cell 

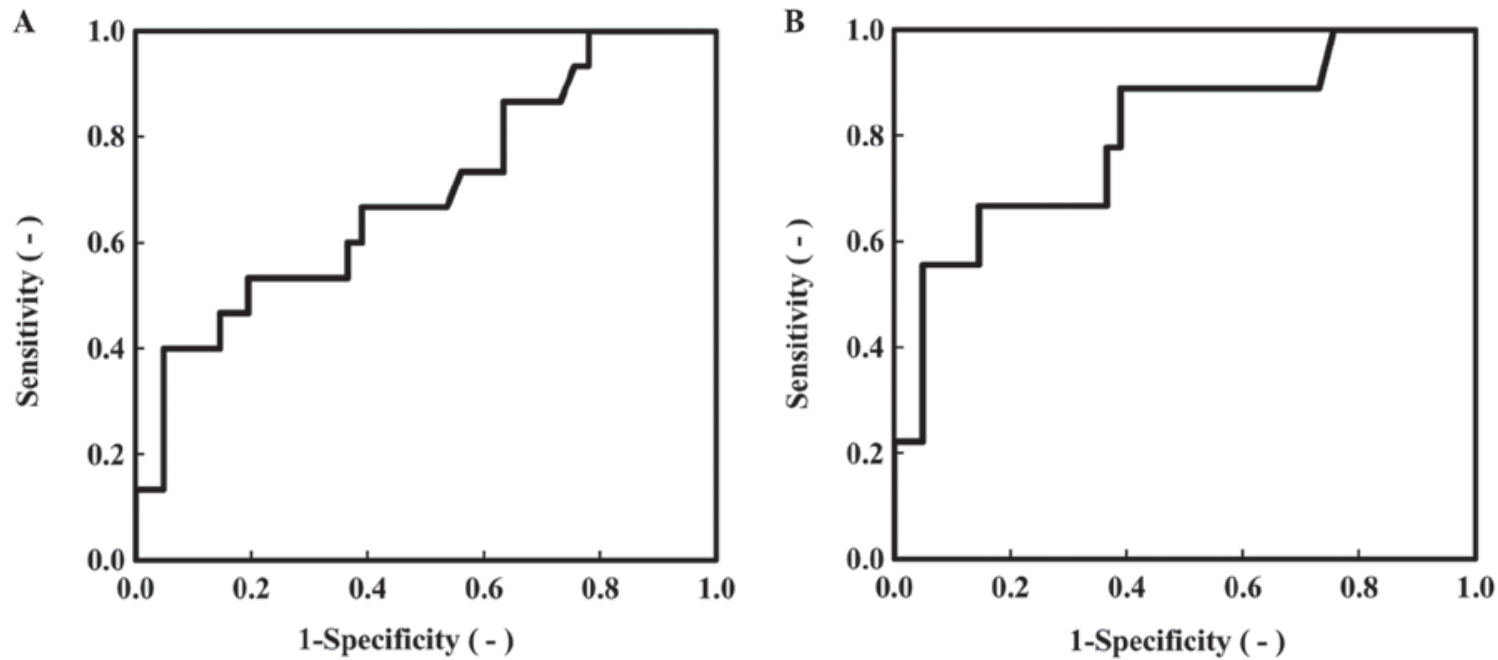

Figure 3. (A) Receiver operating characteristic (ROC) curve for serum angiopoietin-like protein 2 (ANGPTL2) in colorectal cancer (CRC) patients with stage 0 -III disease $(n=15)$. The area under the curve (AUC) was 0.691 [95\% confindence interval (CI): 0.529-0.853] $(\mathrm{P}<0.05)$. (B) ROC curve for serum ANGPTL2 in CRC patients with stage II-III disease (n=9). The AUC was 0.801 (95\% CI: 0.632-0.970) $(\mathrm{P}<0.05)$.

A

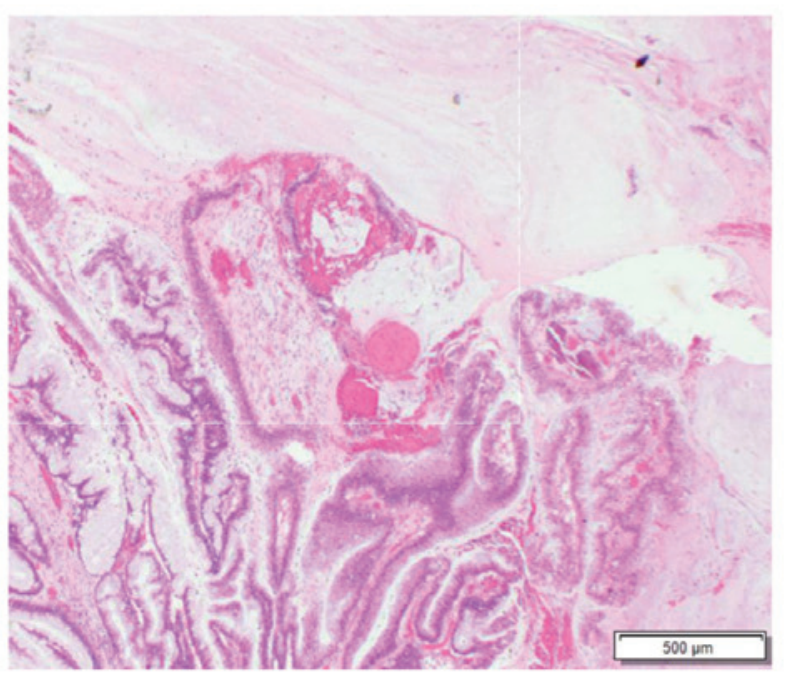

B

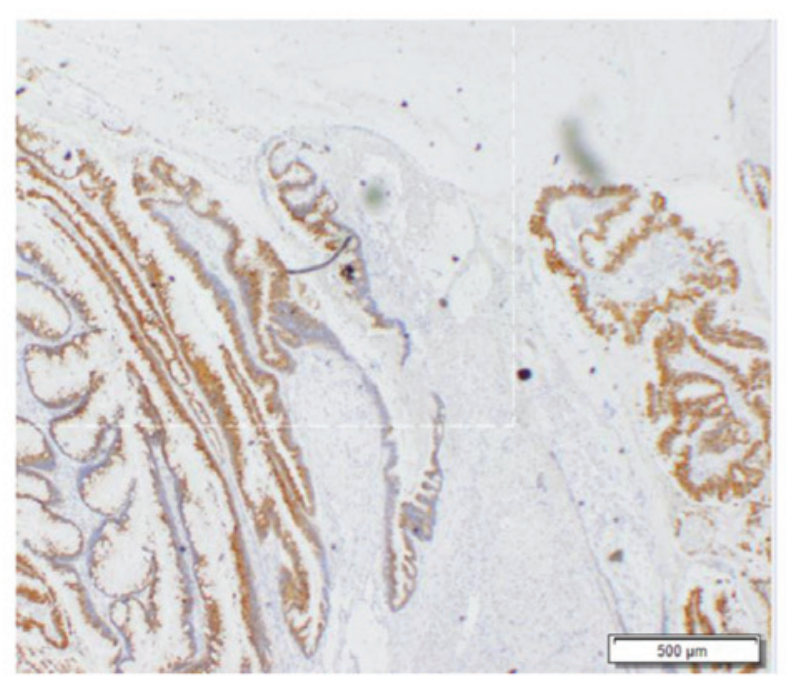

Figure 4. Representative sections from a colorectal cancer patient who exhibited the highest serum angiopoietin-like protein 2 expression levels. The sections from the tumor were (A) stained with hematoxylin and eosin and (B) immunostained with mucin 5AC.

line exhibited the highest level of ANGPTL2 expression. This cell line represents a migratory phenotype with extensive epithelial-to-mesenchymal transition (EMT) and scored the highest in terms of signatures associated with worse overall survival and higher risk of recurrence (12). In our previous study, we reported that the HGC-27 gastric cancer cell line also exhibited a high level of ANGPTL2 expression; this cell line exhibits a low expression level of E-cadherin (13). These results indicate that the expression of ANGPTL2 may be associated with EMT and E-cadherin. However, further studies are required to elucidate the mechanism underlying the enhanced expression of ANGPTL2.

Additionally, we demonstrated that the serum ANGTPL2 levels in CRC patients were higher compared with those in healthy controls. Furthermore, the ROC analysis demonstrated that the diagnostic ability of ANGPTL2 was marginally lower compared with that of the classic biomarker CRP and higher compared with that of CA19-9. These results suggest that simultaneous measurement of ANGPTL2 and the classic biomarkers may reduce the likelihood of overlooking CRC.

It has been reported that colorectal mucinous adenocarcinoma is associated with a poor prognosis. Mucinous adeno carcinoma is often detected at an advanced stage (14). In this study, a CRC patient with mucinous mucinous adenocarcinoma carcinoma exhibited the highest ANGPTL2 level, suggesting that high ANGPTL2 expression may be a predictive biomarker for that type of cancer.

\section{Acknowledgements}

This study was supported in part by the Division of Gene Research, Kagoshima University, the Division of 
Gastrointestinal Surgery, Nanpuh Hospital, the Division of Diagnostic Pathology, Nanpuh Hospital and the Division of Clinical Laboratory, Nanpuh Hospital.

\section{References}

1. Jemal A, Bray F, Center MM, Ferlay J, Ward E and Forman D: Global cancer statistics. CA Cancer J Clin 61: 69-90, 2011.

2. Yoshida N, Yagi N, Naito Y and Yoshikawa T: Safe procedure in endoscopic submucosal dissection for colorectal tumors focused on preventing complications. World J Gastroenterol 16 : $1688-1695,2010$

3. Chapman MA, Buckley D, Henson DB and Armitage NC: Preoperative carcinoembryonic antigen is related to tumour stage and long-term survival in colorectal cancer. Br J Cancer 78 1346-1349, 1998.

4. Kuusela P, Jalanko H, Roberts P, Sipponen P, Mecklin JP, Pitkänen R and Mäkelä O: Comparison of CA 19-9 and carcinoembryonic antigen (CEA) levels in the serum of patients with colorectal diseases. Br J Cancer 49: 135-139, 1984.

5. Hato T, Tabata M and Oike Y: The role of angiopoietin-like proteins in angiogenesis and metabolism. Trends Cardiovasc Med 18: 6-14, 2008.

6. Tabata M, Kadomatsu T, Fukuhara S, et al: Angiopoietin-like protein 2 promotes chronic adipose tissue inflammation and obesity-related systemic insulin resistance. Cell Metab 10 $178-188,2009$.
7. Kadomatsu T, Tabata M and Oike Y: Angiopoietin-like proteins: Emerging targets for treatment of obesity and related metabolic diseases. FEBS J 278: 559-564, 2011.

8. Aoi J, Endo M, Kadomatsu T, et al: Angiopoietin-like protein 2 is an important facilitator of inflammatory carcinogenesis and metastasis. Cancer Res 71: 7502-7512, 2011.

9. Endo M, Nakano M, Kadomatsu T, et al: Tumor cell-derived angiopoietin-like protein ANGPTL2 is a critical driver of metastasis. Cancer Res 72: 1784-1794, 2012.

10. Okada T, Tsukano H, Endo M, et al: Synoviocyte-derived angiopoietin-like protein 2 contributes to synovial chronic inflammation in rheumatoid arthritis. Am J Pathol 176: 2309-2319, 2010

11. Yoshinaga T, Shigemitsu T, Nishimata $\mathrm{H}$, Takei T and Yoshida M: Angiopoietin-like protein 2 is a potential biomarker for gastric cancer. Mol Med Rep 11: 2653-2658, 2015.

12. Christensen J, El-Gebali S, Natoli M, et al: Defining new criteria for selection of cell-based intestinal models using publicly available databases. BMC Genomics 13: 274, 2012.

13. Kuwabara Y, Yamada T, Yamazaki K, Du WL, Banno K, Aoki D and Sakamoto M: Establishment of an ovarian metastasis model and possible involvement of E-cadherin down-regulation in the metastasis. Cancer Sci 99: 1933-1939, 2008.

14. Symonds DA and Vickery AL: Mucinous carcinoma of the colon and rectum. Cancer 37: 1891-1900, 1976. 